\title{
High-pressure nuclear inelastic scattering with backscattering monochromatization
}

\author{
Ilya Sergueev, Konstantin Glazyrin, Markus G. Herrmann, Pavel Alexeev, \\ Hans-Christian Wille, Olaf Leupold, Andrew F. May, Tribhuwan Pandey, \\ Lucas R. Lindsay, Karen Friese and Raphael P. Hermann
}

J. Synchrotron Rad. (2019). 26, 1592-1599

\section{IUCr Journals CRYSTALLOGRAPHY JOURNALS ONLINE}

Copyright (C) International Union of Crystallography

Author(s) of this article may load this reprint on their own web site or institutional repository provided that this cover page is retained. Republication of this article or its storage in electronic databases other than as specified above is not permitted without prior permission in writing from the IUCr.

For further information see http://journals.iucr.org/services/authorrights.html 
JOURNAL OF SYNCHROTRON RADIATION

ISSN 1600-5775

Received 15 October 2018

Accepted 21 June 2019

Edited by D. A. Reis, SLAC National Accelerator Laboratory, USA

Keywords: nuclear inelastic scattering; high pressure; backscattering; nuclear resonance scattering; density of phonon states.

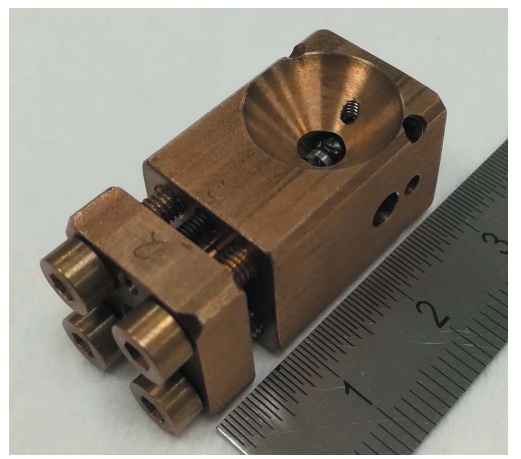

C 2019 International Union of Crystallography

\section{High-pressure nuclear inelastic scattering with backscattering monochromatization}

\author{
Ilya Sergueev, ${ }^{a *}$ Konstantin Glazyrin, ${ }^{a}$ Markus G. Herrmann, ${ }^{b}$ Pavel Alexeev, ${ }^{a}$ \\ Hans-Christian Wille, ${ }^{a}$ Olaf Leupold, ${ }^{a}$ Andrew F. May, ${ }^{c}$ Tribhuwan Pandey, \\ Lucas R. Lindsay, ${ }^{\mathrm{c}}$ Karen Friese ${ }^{\mathrm{b}}$ and Raphael P. Hermann ${ }^{\mathrm{c} *}$
}

${ }^{a}$ Deutsches Elektronen-Synchrotron DESY, D-22607 Hamburg, Germany, ${ }^{\mathbf{b}}$ Jülich Centre for Neutron Science-2/Peter Grünberg Institut-4, Forschungszentrum Jülich GmbH, D-52425 Jülich, Germany, and ${ }^{\mathbf{c}}$ Materials Science and Technology Division, Oak Ridge National Laboratory, Oak Ridge, TN 37831, USA. *Correspondence e-mail: ilya.sergeev@desy.de, hermannrp@ornl.gov

The capability to perform high-pressure low-temperature nuclear inelastic scattering on ${ }^{125} \mathrm{Te}$ and ${ }^{121} \mathrm{Sb}$ with a sapphire backscattering monochromator is presented. This technique was applied to measure nuclear inelastic scattering in $\mathrm{TeO}_{2}$ at pressures up to $10 \mathrm{GPa}$ and temperatures down to $25 \mathrm{~K}$. The evaluated partial Te densities of phonon states were compared with theoretical calculations and with Raman scattering measured under the same conditions. The high-pressure cell developed in this work can also be used for other techniques at pressures up to at least $100 \mathrm{GPa}$.

\section{Introduction}

Nuclear inelastic scattering (NIS) is a technique for studying vibrational modes and phonons in order to investigate the thermodynamic, vibrational and elastic properties of solids (Seto et al., 1995; Sturhahn et al., 1995; Chumakov \& Sturhahn, 1999). Because it utilizes nuclear resonant absorption for particular isotopes, it provides element-specific insights for compounds possessing those isotopes. The majority of NIS studies are performed with ${ }^{57} \mathrm{Fe}$ and other isotopes with nuclear resonance energies below $30 \mathrm{keV}$, such as ${ }^{119} \mathrm{Sn},{ }^{151} \mathrm{Eu}$, ${ }^{149} \mathrm{Sm}$ and ${ }^{161} \mathrm{Dy}$. The development of sapphire backscattering monochromators (BSMs; Shvyd'ko \& Gerdau, 1999; Wille et al., 2006, 2010a; Sergueev et al., 2011) has opened a route to applying NIS to isotopes with resonance energies above $30 \mathrm{keV}$, namely ${ }^{121} \mathrm{Sb},{ }^{125} \mathrm{Te}$ and ${ }^{129} \mathrm{Xe}$ (Klobes et al., 2013). This is beneficial for the study of the many novel functional materials with antimony and tellurium constituent elements. Examples include $\mathrm{Fe}(\mathrm{Se}, \mathrm{Te})$-based high-temperature superconductors (Mizuguchi \& Takano, 2010; Ksenofontov et al., 2010), Ge-Sb-Te-based phase-change materials (Wuttig \& Yamada, 2007; Matsunaga et al., 2011), and Te- or Sb-based thermoelectrics and topological insulators: binary compounds, e.g. $\mathrm{PbTe}$ (Bauer Pereira et al., 2013), $\mathrm{Bi}_{2} \mathrm{Te}_{3}$ and $\mathrm{Sb}_{2} \mathrm{Te}_{3}$ (Bessas et al., 2012), or complex nanostructured compounds, such as $\left(\mathrm{AgSbTe}_{2}\right)_{x}(\mathrm{PbTe})_{1-x}$ (Snyder \& Toberer, 2008). The functional, structural and thermal properties of these materials are related to their phonon structure and resulting phenomena like electron-phonon coupling and anharmonicity.

One of the advantages of NIS is its applicability at high pressure, up to $1 \mathrm{Mbar}$ (1 bar $=100000 \mathrm{~Pa}$; Zhao et al., 2004). The response of phonon modes to compression provides detailed information on the material's anharmonicity, sound 
velocity and nonlinear lattice behaviour (Zhang et al., 2013; Parshin et al., 2016; Sergueev et al., 2015). Such studies are important for the synthesis and design of new compounds, as the physical properties can be finely tuned and investigated by modifying the interatomic distances under pressure.

The room-temperature Lamb-Mössbauer factor is extremely small for $\mathrm{Sb}$ and $\mathrm{Te}$ within most materials as the nuclear resonance energies for the ${ }^{121} \mathrm{Sb}$ and ${ }^{125} \mathrm{Te}$ nuclear isotopes are quite high at 37.1 and $35.5 \mathrm{keV}$, respectively. This leads to multi-phonon scattering contributions that dominate the inelastic scattering signal and, therefore, prevent the extraction of the one-phonon scattering term and the partial density of phonon states (PDOS). Thus, NIS with these isotopes must be conducted at temperatures generally below $50-100 \mathrm{~K}$, a requirement that also remains valid for high-pressure studies, except at very high pressures (above 50-100 GPa), where the densification of the material ultimately leads to a LambMössbauer factor increase (Simon et al., 2014). However, scientific interest in many applied materials is focused upon moderate pressures (below 20-30 GPa).

Here, we describe a new capability for high-pressure lowtemperature nuclear inelastic scattering, using the $35.5 \mathrm{keV}$ nuclear resonance transition in the ${ }^{125} \mathrm{Te}$ isotope on the Dynamics Beamline P01 at PETRA III (DESY, Hamburg) (Wille et al., 2010b). The experimental setup for high-resolution sapphire backscattering monochromatization and beam focusing at the sample position is described in the next section. The miniaturized panoramic diamond-anvil high-pressure cell, which gives access to pressures up to at least $100 \mathrm{GPa}$, is described in the third section. The fourth section demonstrates the application of the technique to study phonons in $\mathrm{TeO}_{2}$ at pressures up to $10 \mathrm{GPa}$ and temperatures down to $25 \mathrm{~K}$. The last section is devoted to a discussion of possible improvements to the setup and the applicability of the method to the ${ }^{121} \mathrm{Sb}$ nuclear isotope.

\section{Experimental setup}

The challenge of nuclear inelastic scattering with a sapphire BSM is to provide a reasonable energy resolution, $\sim 1 \mathrm{meV}$, as required for meaningful studies of phonons. This resolution at energies of 35-40 keV requires an ultra-high-quality monochromator crystal with a lattice spacing variation $\Delta d / d \leq 10^{-8}$, where $d$ is the lattice parameter. Topographic investigations of sapphire crystals (Sergueev et al., 2011; Jafari et al., 2017) show that such quality can be reached, but so far only over a limited area with a characteristic size of $0.5 \mathrm{~mm}$. This imposes limitations on experimental setups for which the beam size at the BSM position must then be of the order of $0.5 \mathrm{~mm}$ or less.

Another parameter influencing the energy resolution is the angular offset from exact backscattering, $\Delta \theta=\pi / 2-\theta$, where $\theta$ is the Bragg angle. The energy resolution near the exact backscattering is defined as $\Delta E / E=\left[(\Phi \Delta \theta)^{2}+\left(\Delta E_{0} / E\right)^{2}\right]^{1 / 2}$, where $\Delta E_{0}$ is the energy resolution at exact backscattering $(\theta=$ $\pi / 2$ ) and $\Phi$ is the divergence of the incoming beam in the scattering plane. For typical values of $E=35 \mathrm{keV}, \Phi=20 \mu \mathrm{rad}$ (horizontal beam divergence) and $\Delta E_{0}=0.6 \mathrm{meV}$, the effect of the angular offset on the energy resolution, $\Phi \Delta \theta$, becomes comparable with $\Delta E_{0} / E$ at $\Delta \theta=0.05^{\circ}$. Thus, in order to avoid energy-resolution broadening, $\Delta \theta$ must be less than $0.05^{\circ}$. This limits the gap at the sample position between the beams incident on and reflected from the BSM to $\sim 35 \mathrm{~mm}$, assuming a $20 \mathrm{~m}$ longitudinal distance between the BSM and the sample. This restriction cannot be alleviated by decreasing $\Phi$ through collimation of the incoming beam because of the requirement to have a small size for the beam impinging on the monochromator crystal, as discussed above. Thus, the sample environment, which is ultimately the pressure cell, must be small in size, at least in the scattering plane, so as to accommodate the two beams.

The small gap between the incident and reflected beams also limits the choice for optical devices that focus the beam at the sample position. Previously, Kirkpatrick-Baez mirrors were used to focus the beam in a high-pressure roomtemperature NIS experiment with backscattering monochromatization (Simon et al., 2014). The large size of the device installed $\sim 1 \mathrm{~m}$ before the sample requires a large angular offset $\Delta \theta$ and, therefore, led to a significant increase in the energy resolution. Here, we used compound refractive lenses (CRLs) as focusing devices, which provide a reasonable beam size at the sample position over the studied pressure range and do not add additional restrictions on the beam path.

The experimental setup employed for our study on beamline P01 at PETRA III is shown in Fig. 1 . At $35.5 \mathrm{keV}$ the undulator beam has a source size of $25 \mu \mathrm{m} \times 330 \mu \mathrm{m}$ (FWHM) and an angular divergence of $10 \mu \mathrm{rad} \times 20 \mu \mathrm{rad}$ (FWHM), vertically and horizontally $(\mathrm{V} \times \mathrm{H})$, respectively. The beam was monochromated to a bandwidth of $5 \mathrm{eV}$ using an $\mathrm{Si}(111)$ double-crystal high-heat-load monochromator (DCM). The beam was collimated in the vertical direction to a size of $0.5 \mathrm{~mm}$ using the curvature of the DCM crystals and optimizing the position of the beam on those crystals.

The 34 cylinder parabolic Be CRLs $\left(\mathrm{CRL}_{1}\right)$, with radii of $0.5 \mathrm{~mm}$ and geometric apertures of $1 \mathrm{~mm}$, were installed $67 \mathrm{~m}$ from the source in order to focus the beam in the horizontal direction. The beam size at the BSM, $88 \mathrm{~m}$ from the source, was $\sim 0.5 \mathrm{~mm} \times 0.6 \mathrm{~mm}(\mathrm{~V} \times \mathrm{H})$.

The sapphire crystal, installed in a nitrogen gas-flow cryostat, reflected the beam in the vertical direction with an angular offset of $\Delta \theta=0.029^{\circ}$ from exact backscattering. The

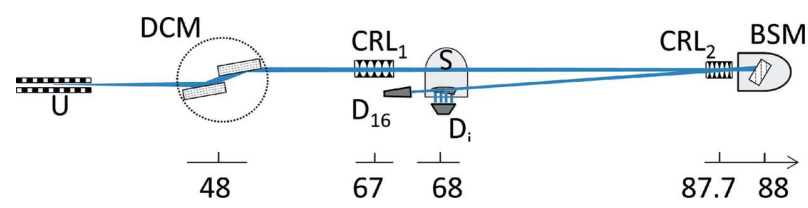

Figure 1

The experimental setup for an NIS experiment on the P01 beamline at PETRA III with undulators (U), a high-heat-load double-crystal monochromator (DCM), horizontal cylinder parabolic Be compound refractive lenses $\left(\mathrm{CRL}_{1}\right)$, the sample in the cryostat (S), rotationally parabolic Be compound refractive lenses $\left(\mathrm{CRL}_{2}\right)$, the sapphire backscattering monochromator (BSM) crystal in the nitrogen gas-flow cryostat and avalanche photodiode detectors $\left(\mathrm{D}_{\mathrm{i}}, \mathrm{D}_{16}\right)$. The bottom scale shows the distances in metres from the source to the optical elements. 
crystal temperature was controlled with millikelvin precision (Alexeev et al., 2016). The position of the beam on the crystal was optimized to achieve the best possible energy resolution.

Nineteen rotationally parabolic Be CRLs $\left(\mathrm{CRL}_{2}\right)$, with radii of $1 \mathrm{~mm}$ ( $2 \mathrm{~mm}$ geometrical aperture), were installed $0.3 \mathrm{~m}$ upstream of the BSM. The beam passes through these CRLs in two directions with an offset of $0.3 \mathrm{~mm}$, comparable with the beam size and smaller than the CRL radius. This setup provides an effective duplication of the focusing power without requiring additional lenses and achieves a beam size of $140 \mu \mathrm{m} \times 230 \mu \mathrm{m}(\mathrm{V} \times \mathrm{H})$ at the sample position.

The nuclear resonance signal was separated in time from electronic X-ray fluorescence and scattering, and measured using two Si avalanche photodiode (APD) systems. An array of 16 APD detectors $\left(D_{16}\right)$ was installed in the forward direction, with a separation between the electronic scattering and the delayed nuclear resonance scattering signal of $2.5 \mathrm{~ns}$ and an absorption efficiency to $35.5 \mathrm{keV}$ of $\sim 25 \%$. Two $10 \mathrm{~mm} \times 10 \mathrm{~mm}$ APD detectors $\left(\mathrm{D}_{\mathrm{i}}\right)$ were installed on both sides, horizontally, close to the sample and detecting mainly the $27.4 \mathrm{keV}$ internally converted nuclear fluorescence that follows the nuclear inelastic absorption process. The separation between the electronic scattering and the delayed nuclear resonance signal for the latter detectors was $5 \mathrm{~ns}$. The absorption efficiency of each detector was $\sim 7 \%$.

The total flux of the beam between the sapphire BSM and the sample was monitored by a $100 \mu$ m-thick Si APD detector which indicated a total flux of $1 \times 10^{8}$ photons $^{-1}$ at the sample position.

The ${ }^{125} \mathrm{Te}$ nuclear resonance energy was reached using the (9 $1 \overline{10} 68$ ) sapphire reflection (in the hexagonal notation). The nuclear resonance signal of the sapphire crystal was observed at a temperature of 217.5 (1) K. The temperatureenergy conversion parameter was $0.149 \mathrm{meV} \mathrm{mK}^{-1}$ as confirmed by measurement of the NIS spectra on elemental

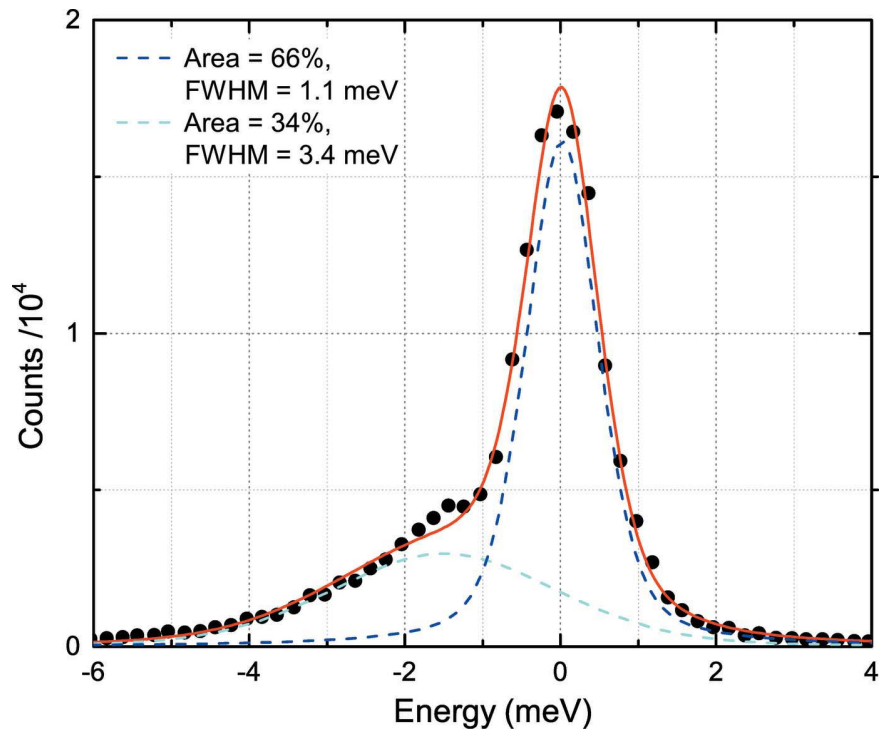

Figure 2

The instrumental function of the sapphire BSM measured with the nuclear forward scattering of ${ }^{125} \mathrm{Te}$. The red curve shows a fit to the data with a combination of two pseudo-Voigt profiles.
Te. The sapphire BSM instrumental function measured by $\mathrm{D}_{16}$ is shown in Fig. 2 and corresponds to a FWHM of $1.25 \mathrm{meV}$, whereas a $0.6 \mathrm{meV}$ resolution is expected in theory. The function contains an additional satellite peak resulting from defects in the sapphire crystal. Fitting the data by two pseudoVoigt functions, we obtain areas of $66 \%$ and $34 \%$ for the main and satellite peaks, with FWHMs of 1.1 and $3.4 \mathrm{meV}$, respectively.

\section{Design of the high-pressure cell}

The main requirement for the high-pressure cell used in the NIS experiment is the small distance required between the sample and the detectors to increase the solid angle for the $10 \mathrm{~mm} \times 10 \mathrm{~mm}$ APD detectors that collect the nuclear fluorescence flux. A system with similar purpose that includes a miniature panoramic diamond anvil cell (DAC) in a cryostat was developed on beamline 3-ID of the APS (Bi et al., 2015; Zhao et al., 2017). However, that system would not fit the backscattering setup reported here because of the requirement to have the path for the incident beam close to the DAC.

Within this work we designed and utilized a new compact piston-cylinder DAC with typical dimensions of $15 \mathrm{~mm} \times$ $15 \mathrm{~mm} \times 30 \mathrm{~mm}$ in the assembled state (see Fig. 3). The technical drawings of the cell were produced at the Jülich Centre for Neutron Science-2, Forschunszentrum Jülich GmbH, Germany (JCNS-2). The cell body (piston-cylinder design), the seats supporting the diamonds, and the screws were manufactured from $\mathrm{BeCu}$ alloy at the Bayerisches Geoinstitut, Bayreuth University, Germany.

The distances from the sample position to the three closest faces are $7.5,7.5$ and $8 \mathrm{~mm}$. For compression, the design employs four M3 screws. The cell has several apertures, allowing the incident beam to reach the sample position either in the geometry parallel to the compression axis, i.e. through

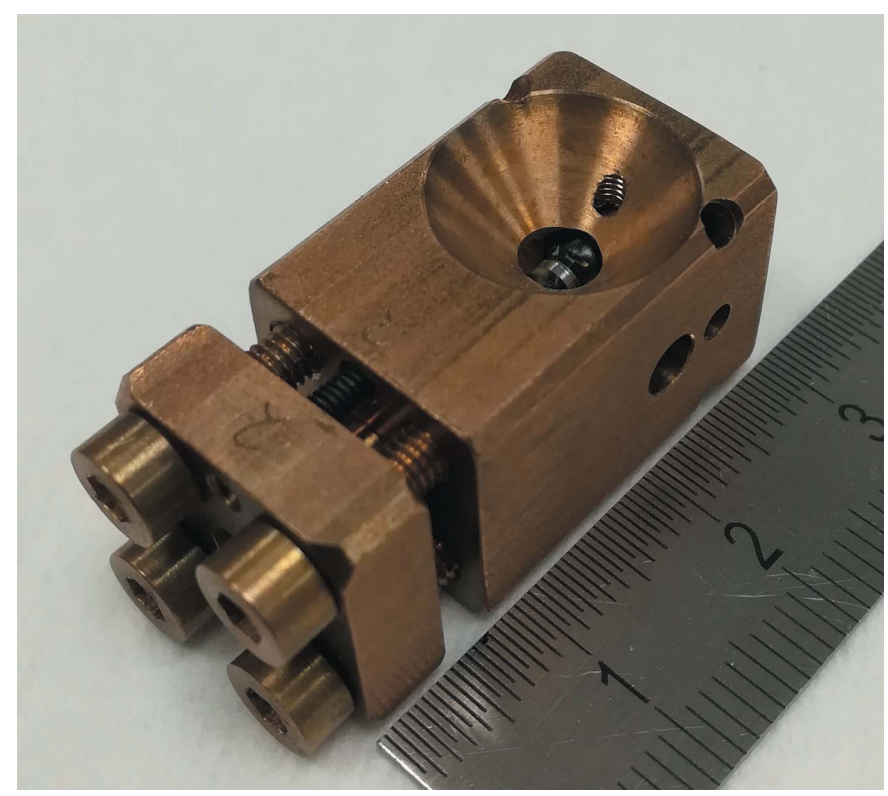

Figure 3

An image of the piston-cylinder DAC employed in this work. 
the diamonds, or perpendicular to the compression axis, i.e. through an X-ray transparent gasket. For the purpose of NIS signal detection we also implemented a conical aperture of $84^{\circ}$ on opposing sides of the cell, namely, perpendicular to the compression axis. The opening created by the intersection of the latter aperture with the inner cylinder enables access for installation of X-ray transparent gaskets $(4-5 \mathrm{~mm}$ in diameter), as well as sample or pressure-standard loading on the anvil attached to the cell body (cylinder). The rectangular shape of the cell helps us to reach a compromise in several desired features: (i) a minimum sample-to-detector distance, (ii) easy mounting onto flat surfaces, (iii) the possibility to stack several cells, and (iv) a low thermal mass.

While in the current state we cannot increase pressure by means of a gas membrane, this option is foreseen and will be implemented in the future, enabling pressure changes at low temperature.

In addition to the application of the cell to NIS experiments, the chosen design also allows for easy expansion in terms of other available methodologies, such as X-ray emission spectroscopy, X-ray absorption near-edge structure (XANES), extended X-ray absorption fine structure (EXAFS), X-ray diffraction (radial geometry) and Raman scattering.

The DAC stage was mounted in a closed-cycle cryostat as shown in Fig. 4. The cryostat cap was designed and manufactured at JCNS-2. Two APD detectors were installed on the sides in front of the Kapton windows at a distance of $10 \mathrm{~mm}$

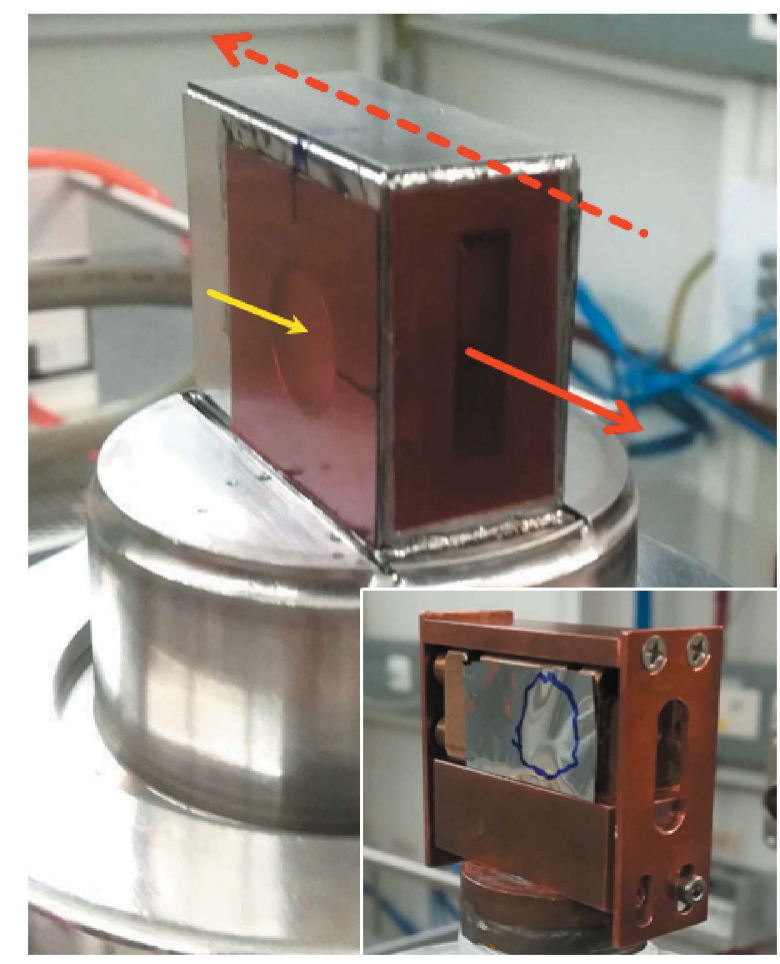

Figure 4

The setup of the high-pressure DAC stage, together with a cryostat without (inset) and with a cryostat cap. Red arrows show the beam incident on the BSM (dashed arrow) and the reflected beam (solid arrow) with a gap of $20 \mathrm{~mm}$ between the two beams. The yellow arrow indicates the Kapton windows in front of which the APD detector was installed. from the sample. The beam incident on the BSM passes $20 \mathrm{~mm}$ above the reflected beam.

The closed-cycle cryostat produces vibrations of the order of $20-40 \mu \mathrm{m}$ in the directions along and perpendicular to the beam. They lead to jitter of the beam at the sample position but this is not important for this setup due to the beam size of $140 \mu \mathrm{m} \times 230 \mu \mathrm{m}$.

The lowest temperature of the closed-cycle cryostat cold finger is $\sim 10 \mathrm{~K}$. The lowest temperature of the sample is defined by the thermal gradient between the cryostat cold finger and the DAC stage and by the radiation heat-exchanged through the Kapton windows. The thermal contact between the DAC stage and the cold finger in the current work allowed the sample to reach $25 \mathrm{~K}$, as confirmed by the detailed balance of the NIS spectra, see below.

\section{High-pressure nuclear inelastic scattering with ${ }^{125} \mathrm{TeO}_{2}$}

To demonstrate the capabilities of the above-described method and equipment, we measured high-pressure NIS on ${ }^{125} \mathrm{TeO}_{2} \cdot \mathrm{TeO}_{2}$ has peculiar nonlinear optical and lattice dynamics properties and is utilized for example in acoustooptics (Kondratyuk et al., 1987; Thomas, 1988). At ambient pressure, three polymorphs, $\alpha, \beta$ and $\gamma$, are known for $\mathrm{TeO}_{2}$. In this study we have utilized $\alpha-\mathrm{TeO}_{2}$, paratellurite (space group $P 4_{1} 2{ }_{1} 2$, tetragonal phase) (Lindqvist, 1968; Kondratyuk et al., 1987), which is formed by corner-sharing $\mathrm{TeO}_{4}$ trigonal bipyramids. Under compression it transforms to an orthorhombic phase (space group $P 2_{1} 2_{1} 2_{1}$ ) at $0.9 \mathrm{GPa}$ via a secondorder transition caused by acoustic mode softening (Worlton \& Beyerlein, 1975; Liu, 1987).

Fine-grained polycrystalline $\mathrm{TeO}_{2}$, prepared by oxidation of $95 \%{ }^{125} \mathrm{Te}$-enriched elemental tellurium, was loaded into a $200 \mu \mathrm{m}$ hole of the Be gasket, without a pressure-transmitting medium, together with ruby chips used as pressure markers. The initial sample thickness was $\sim 60 \mu \mathrm{m}$. The gasket was mounted into the DAC equipped with $400 \mu \mathrm{m}$ culet diameter diamond anvils. Measurements were performed at 5.5 (3) and 8.4 (3) GPa and low temperatures.

Pressure was applied at room temperature before installation of the DAC stage into the cryostat and monitored by the ruby fluorescence at room temperature before and after the NIS studies. We observed minor pressure changes within $0.3 \mathrm{GPa}$ between the before and after measurements due to cycling of the temperature in between and time-dependent pressure creep. Cooling from room temperature to low temperature may increase the pressure. However, additional compression results in irreversible gasket deformation, and the process of the pressure increase upon cooling is usually also irreversible. Thus, we would see a residual increase in pressure after warming up. Since our starting and final pressures, namely, before and after cooling, are within a range of $0.3 \mathrm{GPa}$, the pressure at the low temperature would be within $0.3 \mathrm{GPa}$ of the pressure before cooling.

In addition to the NIS experiment, nuclear forward scattering (NFS) and Raman studies were performed. We used the 


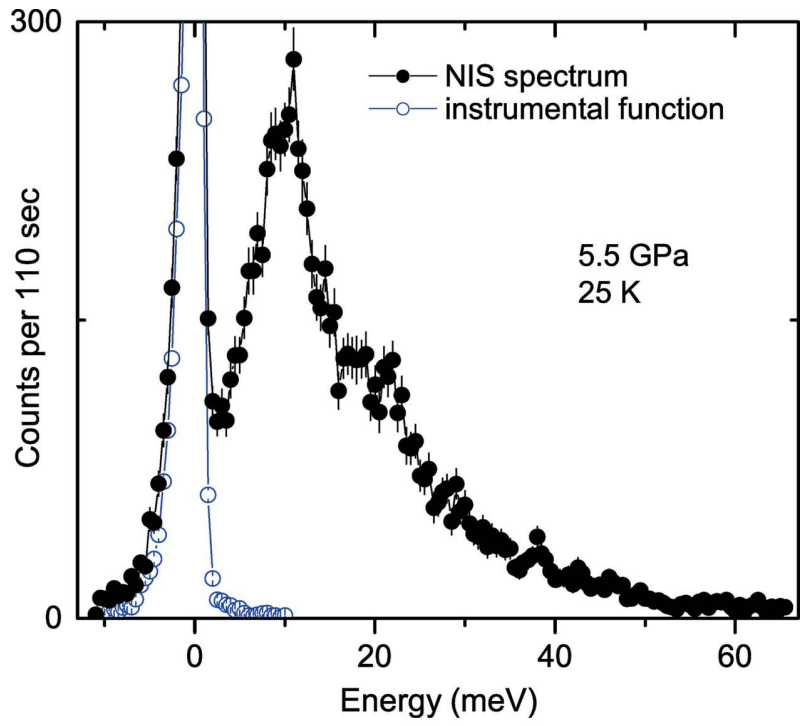

Figure 5

The ${ }^{125} \mathrm{Te}$ NIS spectrum of $\mathrm{TeO}_{2}$ measured at 5.5 (3) GPa and 25 (1) K. The blue open circles represent the instrumental function scaled down by a factor of 5 .

Raman system installed on the PETRA III Extreme Conditions Beamline P02.2 and Extreme Conditions Science Infrastructure (ESCI) (Liermann et al., 2015). We employed a $532 \mathrm{~nm}$ wavelength laser with a power of $\sim 40 \mathrm{~mW}$ focused on the sample, an Andor Shamrock SR-500i-A spectrometer equipped with a DU940P-BV CCD camera and a grating with a period of 1800 lines $\mathrm{mm}^{-1}$.

The ${ }^{125} \mathrm{Te}$ NIS spectrum of $\mathrm{TeO}_{2}$ at 5.5 (3) $\mathrm{GPa}$ is shown in Fig. 5. The total measurement time was $\sim 5 \mathrm{~h}$. The detailed thermal balance of the negative and positive parts of the spectrum evaluated with Bose-Einstein statistics yields a sample temperature of 25 (2) K.

The NFS time spectra measured on the sample at ambient pressure, $296 \mathrm{~K}$ and under the conditions of the NIS measurements are shown in Fig. 6. A model using a quadrupole hyperfine interaction and the sample thickness gives reasonable agreement with the measurements. The quadrupole splitting decreases slightly with pressure as shown in the inset to Fig. 6. The ambient-pressure value of the quadrupole splitting agrees well with the results reported by Jung \& Triftshäuser (1968) and Klobes et al. (2014).

The ${ }^{125}$ Te PDOS evaluated from the NIS spectra are shown in Fig. 7. For the purpose of this discussion, we reproduce here also the PDOS previously measured at ambient pressure (Jafari, 2016).

In order to take into account the asymmetry of the instrumental function which gives an artificial shift of the PDOS peaks, the data treatment includes a deconvolution of the NIS spectra with the instrumental function and convolution by a Gaussian with a $2 \mathrm{meV}$ FWHM. The convolution with a Gaussian was used here to suppress high-frequency noise appearing due to the deconvolution. Because of the fast decay of the Gaussian tails, the artificial $2 \mathrm{meV}$ frequency oscillations are present in the spectra.

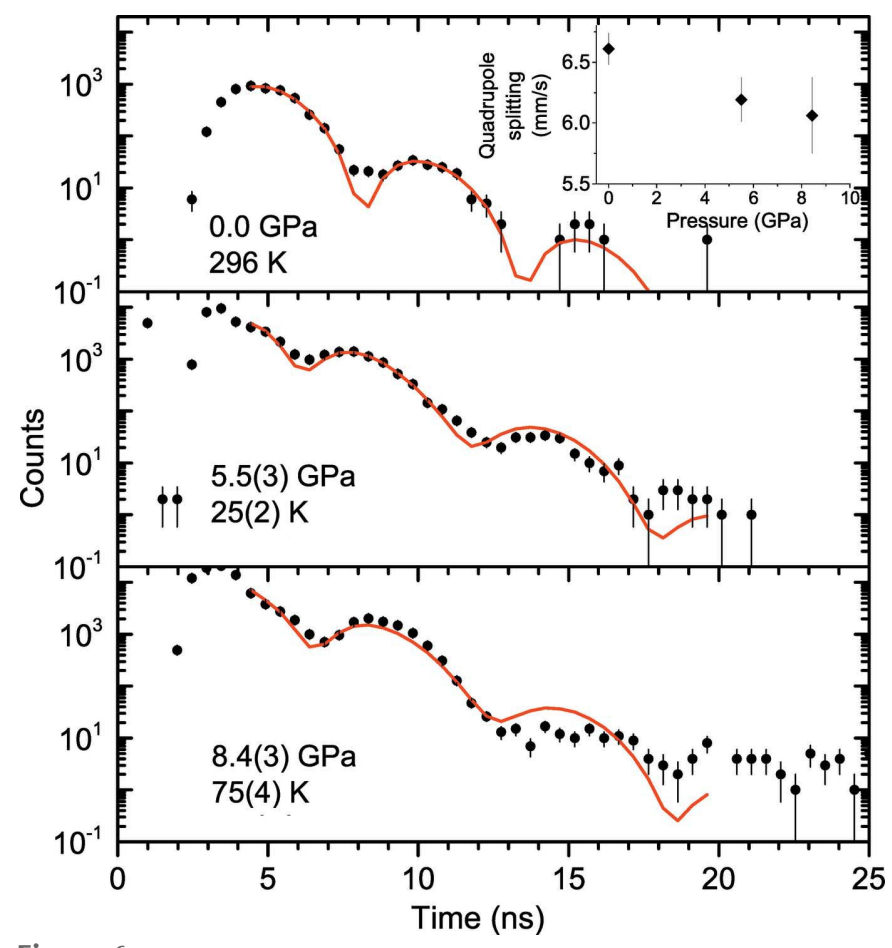

Figure 6

${ }^{125} \mathrm{Te}$ NFS time spectra of $\mathrm{TeO}_{2}$ measured at ambient pressure and room temperature (top), and at 5.5 (3) and 8.4 (3) GPa (centre and bottom), together with the NIS measurements. The red curves show fits using the quadrupole hyperfine interaction and the sample thickness.

The Raman peaks evaluated from the Raman spectra measured on the sample at room temperature and the corresponding pressures are shown in Fig. 7 together with the PDOS. The Raman spectra were filtered at $130 \mathrm{~cm}^{-1}$ by a notch filter. The positions of the Raman peaks here agree well with the results reported by Kourouklis \& Jayaraman (1992).

The PDOS at ambient pressure includes a pronounced peak at $10 \mathrm{meV}$, which corresponds to the van Hove singularity of the acoustic phonon branches, as well as a set of optical phonon peaks with well resolved energies at 19-24 and $27 \mathrm{meV}$. Compression leads to hardening of the PDOS and the Raman peaks in a similar manner. The effect of the compression on lattice dynamics parameters, such as the Lamb-Mössbauer factor, mean sound velocity and displacement parameters, are shown in Table 1 . The phonon hardening is nearly proportional to the applied pressure. The parameters and peak positions change by $\sim 8 \%$ at 5.5 (3) GPa and by $\sim 12 \%$ at 8.4 (3) GPa relative to those at ambient pressure.

The evolution of the acoustic part of the PDOS under compression is seen in the inset to Fig. 7 where PDOS $/ E^{2}$ is shown. The data at high pressure show a flat behaviour with straightforward extrapolation of the Debye level up to $0 \mathrm{meV}$, which is used to calculate the mean sound velocity. On the other hand, the ambient-pressure PDOS shows peaks in $\mathrm{PDOS} / E^{2}$ at 3 and $9 \mathrm{meV}$ which are due to the very soft phonon modes in the ambient-pressure phase. Under these conditions, the extrapolation of the Debye level done here, leading to a mean sound velocity of 2.2 (1) $\mathrm{km} \mathrm{s}^{-1}$, could be debatable. A mean sound velocity of $1.91 \mathrm{~km} \mathrm{~s}^{-1}$ was obtained 
Table 1

Parameters obtained from the PDOS of $\mathrm{TeO}_{2}$ measured at the indicated pressures $P$ and temperatures $T$ : the Lamb-Mössbauer factor $f_{\mathrm{LM}}$, the mean sound velocity $\langle v\rangle$, and the Te atomic displacement parameters $U_{\text {eq }}$ calculated for $T=0 \mathrm{~K}$ and $T=300 \mathrm{~K}$ assuming harmonic phonon behaviour (negligible PDOS change with temperature); $U_{\mathrm{eq}}^{\text {th }}$ shows the Te atomic displacement parameters obtained from theoretical calculations.

\begin{tabular}{|c|c|c|c|c|c|c|c|}
\hline \multirow{2}{*}{$\begin{array}{l}P \\
(\mathrm{GPa})\end{array}$} & \multirow{2}{*}{$\begin{array}{l}T \\
(\mathrm{~K})\end{array}$} & \multirow[b]{2}{*}{$f_{\mathrm{LM}}$} & \multirow{2}{*}{$\begin{array}{l}\langle v\rangle \\
\left(\mathrm{km} \mathrm{s}^{-1}\right)\end{array}$} & \multicolumn{2}{|c|}{$U_{\text {eq }}\left(\mathrm{pm}^{2}\right)$} & \multicolumn{2}{|c|}{$U_{\mathrm{eq}}^{\mathrm{th}}\left(\mathrm{pm}^{2}\right)$} \\
\hline & & & & $0 \mathrm{~K}$ & $300 \mathrm{~K}$ & $0 \mathrm{~K}$ & $300 \mathrm{~K}$ \\
\hline 0.0 & $20(4)$ & $0.645(6)$ & $2.20(10)$ & $12.4(3)$ & 81.3 & 12.40 & 81.48 \\
\hline $5.5(3)$ & $25(2)$ & $0.665(3)$ & $2.38(7)$ & $11.4(2)$ & 66.3 & 11.06 & 62.88 \\
\hline $8.4(3)$ & $75(4)$ & $0.567(3)$ & $2.54(5)$ & $11.1(2)$ & 61.2 & 10.62 & 57.41 \\
\hline
\end{tabular}

by Ogi et al. (2004), leading to the significantly higher Debye level shown in the inset to Fig. 7. The increase in the Debye level is most probably related to the phase transition and a subsequent change in the acoustic modes.

The measurements were complemented by calculations of the phonon dispersions and corresponding PDOS (Fig. 7) of orthorhombic $\mathrm{TeO}_{2}$ at $P=0,5.5$ and $8.4 \mathrm{GPa}$ from density functional theory as implemented in VASP (Kresse \& Furthmüller, 1996). Calculations were derived from the

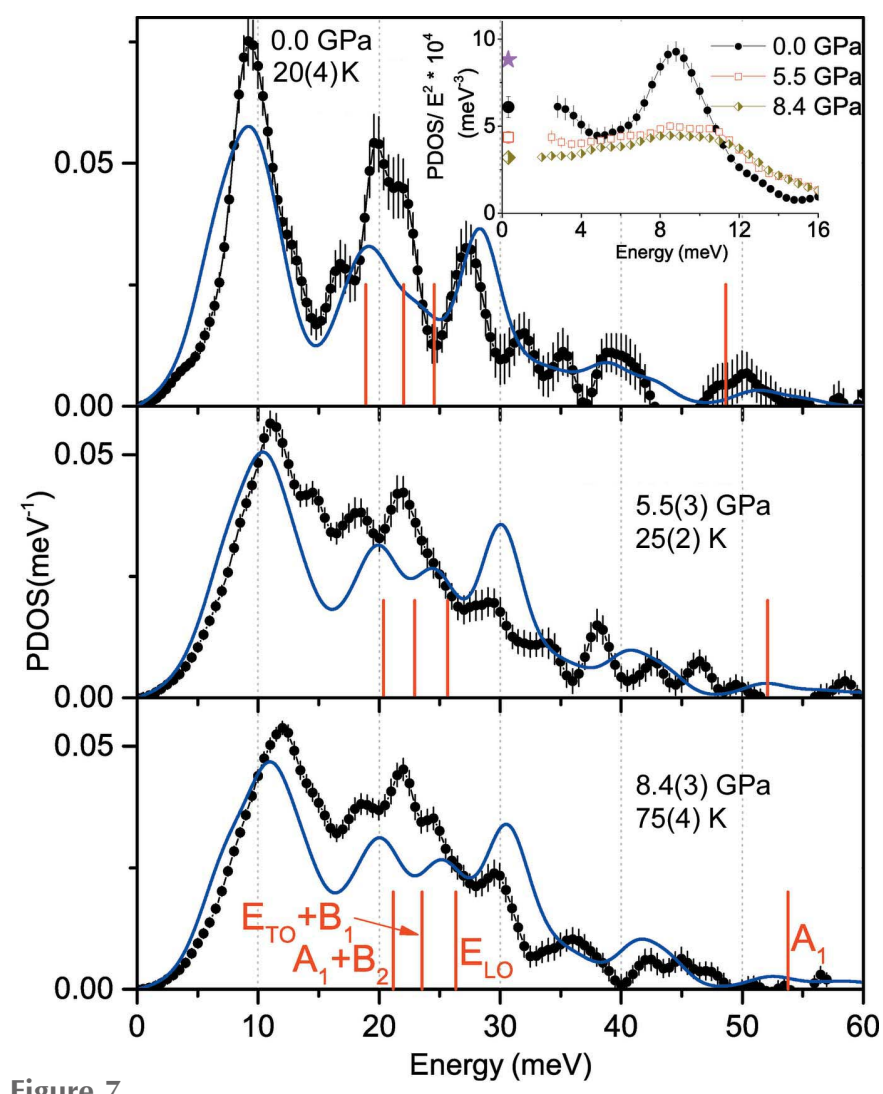

The $\mathrm{Te}$ PDOS of $\mathrm{TeO}_{2}$ measured at the indicated pressures and temperatures. The blue curves show the calculated Te PDOS of orthorhombic $\mathrm{TeO}_{2}$ at the corresponding pressures. The red vertical lines denote the positions of the Raman peaks measured on the same sample at room temperature and the corresponding pressures. The inset shows the reduced PDOS (PDOS $\left./ E^{2}\right)$, with the points at zero energy denoting the extrapolated Debye levels. The magenta star shows the Debye level calculated using the mean sound velocity obtained by Ogi et al. (2004).
PBEsol exchange/correlation functional (Perdew et al., 2008) and PAW pseudopotentials (six valence electrons for each element) (Blöchl, 1994; Kresse \& Joubert, 1999). The orthorhombic $\mathrm{TeO}_{2}$ structure was relaxed (forces $<10^{-3} \mathrm{eV} \AA^{-1}$ ) using a $k$-point grid $4 \times 2 \times 4$ to give optimized lattice parameters $a=5.33, b=11.81$ and $c=5.70 \AA$, in reasonable agreement with measured values (Ito \& Sawada, 1940). To simulate pressure, this ground-state structure was hydrostatically compressed and the atomic positions relaxed to a new equilibrium. Within the pressure range investigated here, $a$ decreased monotonically with pressure (as expected), while $b$ and $c$ decreased initially and then increased slightly upon further increasing the pressure: $P=5.5 \mathrm{GPa}$ gave $a=4.99$, $b=11.47$ and $c=5.67 \AA ; P=8.4$ GPa gave $a=4.80, b=11.48$ and $c=5.69 \AA$.

For all cases, interatomic force constants (necessary for calculations of phonons and related properties) were obtained using the finite displacement method in the PHONOPY package (Togo \& Tanaka, 2015) using $3 \times 2 \times 3$ supercells (432 atoms) and $\Gamma$-point-only calculations. The PDOS was calculated on a dense $q$-point mesh and using Gaussian broadening with a $2 \mathrm{meV}$ width similar to that used to represent the measured data (Fig. 7). We note that PDOS calculations at ambient pressure for different $\mathrm{TeO}_{2}$ polymorphs and including dispersion corrections were presented by Deringer et al. (2014). The general Te PDOS features correlate well with the measured data, namely, the peak positions, and the hardening and broadening of the peaks with pressure. The calculated Te atomic mean-squared displacement parameters at 0 and $300 \mathrm{~K}$ are shown in Table 1 and demonstrate good agreement with the measured values.

\section{Results and discussion}

The experiments performed in this study illustrate the capability to perform ${ }^{125} \mathrm{Te}$ NIS experiments up to $10 \mathrm{GPa}$ at temperatures below $100 \mathrm{~K}$, and, furthermore, they demonstrate that it is possible to obtain a Te PDOS under these conditions.

The setup used for this study can also be utilized to perform ${ }^{121} \mathrm{Sb}$ NIS experiments since the nuclear resonance energy of ${ }^{121} \mathrm{Sb}(37.1 \mathrm{keV})$ is quite close to that of ${ }^{125} \mathrm{Te}$. An Sb NIS study at ambient pressure and low temperature has already been performed on the beamline (Herrmann, 2019).

A disadvantage of the experimental setup presented in this work is the rather large beam size at the BSM and at the sample position. A decrease in the beam size at the BSM to $0.3 \mathrm{~mm} \times 0.3 \mathrm{~mm}$ would significantly improve the energy resolution (Sergueev et al., 2011; Jafari et al., 2017). Furthermore, a decrease in the beam size at the sample position is important in order to increase the pressure range. Samples placed in diamond anvils with a culet size of $400 \mu \mathrm{m}$ can be compressed up to $\sim 20 \mathrm{GPa}$, and with a size of $100 \mu \mathrm{m}$ beyond $100 \mathrm{GPa}$. Moreover, to perform measurements under quasihydrostatic conditions, the sample must be enclosed by a hydrostatic pressure-transmitting medium. In this case, a reasonable sample size must be of the order of $50 \mu \mathrm{m}$ or lower. 


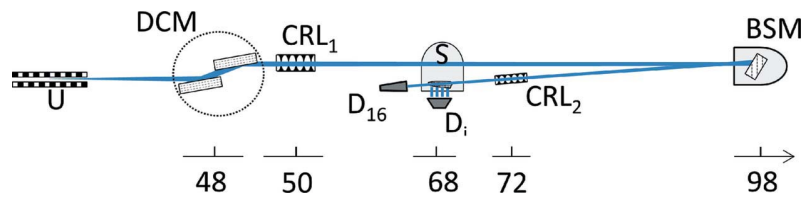

Figure 8

A possible future experimental setup, with the beam focusing at the BSM at the first stage and at the sample (S) at the second stage. The elements here are the same as in Fig. 1. $\mathrm{CRL}_{1}$ and $\mathrm{CRL}_{2}$ are rotationally parabolic $\mathrm{Be}$ compound refractive lenses which focus the beam at the BSM and at the sample, respectively.

One should remark that experiments with beams larger than the sample are still possible, since NIS is an element-specific technique. There would be no signal yield from the Be gasket, pressure medium or diamonds, which do not contain the studied isotope. However, the signal would decrease significantly and thus make data acquisition more time consuming.

An improvement of the setup would require modifying the focusing optics in order to reach a smaller beam size at the BSM and a beam size less than or equal to $50 \mu \mathrm{m}$ at the sample position without having strong absorption effects. The optimal setup with two sets of CRLs is shown in Fig. 8. Here, a first set of rotationally parabolic Be CRLs $\left(\mathrm{CRL}_{1}\right)$ focuses the beam at the BSM with an almost one-to-one ratio of the distances from the CRLs to the X-ray source and to the image. The focal length of the CRL is $25 \mathrm{~m}$. This CRL can be constructed using 15 CRLs with radii of $200 \mu \mathrm{m}$ and geometric apertures of $0.9 \mathrm{~mm}$. The beam size at the BSM will then be the same as the source size, i.e. $25 \mu \mathrm{m} \times 330 \mu \mathrm{m}(\mathrm{V} \times \mathrm{H})$. The second set of rotationally parabolic Be CRLs $\left(\mathrm{CRL}_{2}\right)$ focuses the beam at the sample position with a ratio of 1:6.5, leading to a theoretical beam size at the sample position of $4 \mu \mathrm{m} \times 50 \mu \mathrm{m}(\mathrm{V} \times$ $\mathrm{H})$. This CRL has a focal length of $3.5 \mathrm{~m}$ and can be constructed using 27 CRLs with radii of $50 \mu \mathrm{m}$ and geometric apertures of $0.4 \mathrm{~mm}$. Importantly, due to the large distance between the BSM and $\mathrm{CRL}_{2}$, the distance between the incident and reflected beams would have to be of the order of $20 \mathrm{~mm}$, which provides enough space for the $\mathrm{CRL}_{2}$ installation. The total transmission through both CRLs would be 0.33 , which is comparable with the transmission of 0.5 of the current setup.

A further decrease in the focal size at the sample position is expected after the upgrade of PETRA III to the PETRA IV ultimate storage ring. This would lead to a significant decrease in the horizontal source size which will be comparable with the vertical size. Thus, the beam size at the sample position in the setup discussed above would be less then $10 \mu \mathrm{m}$ in both directions.

\section{Conclusions}

The experimental setup described here demonstrates the principal capability to perform ${ }^{125} \mathrm{Te}$ NIS experiments under compression up to $10 \mathrm{GPa}$ and at temperatures as low as $25 \mathrm{~K}$. The method was applied to obtain partial Te phonon DOS of ${ }^{125} \mathrm{TeO}_{2}$ at 5.5 (3) and 8.4 (3) GPa, which we compared with Raman spectroscopy data and with theoretical calculations. In addition, the evolution of the hyperfine quadrupole splitting under pressure was measured by nuclear forward scattering.

The same method can be also applied to investigate ${ }^{121} \mathrm{Sb}$ nuclear resonance. A possible upgrade of the setup would extend the pressure range where measurements can be performed up to at least $100 \mathrm{GPa}$, covering a wide range of fundamental and applied science applications with ${ }^{125} \mathrm{Te}$ and ${ }^{121} \mathrm{Sb}$ nuclear isotopes.

Here we have also reported the new development of a miniature panoramic diamond-anvil cell and its application to low-temperature NIS studies. Due to the geometry of the backscattering, its small dimensions played a crucial role. At the same time the cell can be used with less complicated setups, e.g. with other nuclear resonances which do not require a BSM, such as ${ }^{57} \mathrm{Fe},{ }^{119} \mathrm{Sn},{ }^{151} \mathrm{Eu},{ }^{149} \mathrm{Sm}$ or ${ }^{161} \mathrm{Dy}$. In addition, the same cell can be used for other synchrotron techniques, like X-ray emission spectroscopy, X-ray absorption near-edge structure (XANES), extended X-ray absorption fine structure (EXAFS) and X-ray diffraction (e.g. in the radial regime, perpendicular to the compression axis). Finally, the optimized dimensions of the cell and the low-friction area between the cylinder and the piston may be advantageous for dynamic compression experiments in the radial geometry at synchrotron and free-electron laser facilities.

\section{Acknowledgements}

We acknowledge DESY (Hamburg, Germany), a member of the Helmholtz Association HGF, for the provision of experimental facilities at PETRA III. We thank Micha Hölzle from the Jülich Centre for Neutron Science-2 for his support in designing the high-pressure cell and for help with modification of the cryostat cap.

\section{Funding information}

This work was supported in part by the Deutsche Forschungsgemeinschaft within the SFB Nanoswitches (SFB 917). Sample synthesis (A. F. May), first-principles phonon calculations (L. Lindsay and T. Pandey), nuclear inelastic scattering and experiment planning (R. P. Hermann) were supported by the US Department of Energy, Office of Science, Basic Energy Sciences, Materials Sciences and Engineering Division.

\section{References}

Alexeev, P., Asadchikov, V., Bessas, D., Butashin, A., Deryabin, A., Dill, F.-U., Ehnes, A., Herlitschke, M., Hermann, R. P., Jafari, A., Prokhorov, I., Roshchin, B., Röhlsberger, R., Schlage, K., Sergueev, I., Siemens, A. \& Wille, H.-C. (2016). Hyperfine Interact. 237, 59.

Bauer Pereira, P., Sergueev, I., Gorsse, S., Dadda, J., Müller, E. \& Hermann, R. P. (2013). Phys. Status Solidi B, 250, 1300-1307.

Bessas, D., Sergueev, I., Wille, H.-C., Perßon, J., Ebling, D. \& Hermann, R. P. (2012). Phys. Rev. B, 86, 224301.

Bi, W., Zhao, J., Lin, J.-F., Jia, Q., Hu, M. Y., Jin, C., Ferry, R., Yang, W., Struzhkin, V. \& Alp, E. E. (2015). J. Synchrotron Rad. 22, 760765.

Blöchl, P. E. (1994). Phys. Rev. B, 50, 17953-17979.

Chumakov, A. \& Sturhahn, W. (1999). Hyperfine Interact. 123/124, 781-808. 
Deringer, V. L., Stoffel, R. P. \& Dronskowski, R. (2014). Cryst. Growth Des. 14, 871-878.

Herrmann, M. G. (2019). Manuscript in preparation.

Ito, T. \& Sawada, H. (1940). Z. Kristallogr. Cryst. Mater. 102, 13.

Jafari, A. (2016). Sapphire Ultraoptics for Sub-meV ${ }^{121} \mathrm{Sb}$ and ${ }^{125} \mathrm{Te}$ Phonon Spectroscopy. Doctoral thesis, Université de Liège, Belgium.

Jafari, A., Sergueev, I., Bessas, D., Klobes, B., Roschin, B. S., Asadchikov, V. E., Alexeev, P., Härtwig, J., Chumakov, A. I., Wille, H.-C. \& Hermann, R. P. (2017). J. Appl. Phys. 121, 044901.

Jung, P. \& Triftshäuser, W. (1968). Phys. Rev. 175, 512-521.

Klobes, B., Barrier, N., Vertruyen, B., Martin, C. \& Hermann, R. P. (2014). Hyperfine Interact. 226, 713-719.

Klobes, B., Desmedt, A., Sergueev, I., Schmalzl, K. \& Hermann, R. P. (2013). Europhys. Lett. 103, 36001.

Kondratyuk, I. P., Muradyan, L. A., Pisarevskii, Y. V. \& Simonov, V. I. (1987). Kristallografiya, 32, 609-617.

Kourouklis, G. A. \& Jayaraman, A. (1992). High. Press. Res. 9, 23-26.

Kresse, G. \& Furthmüller, J. (1996). Comput. Mater. Sci. 6, 15-50.

Kresse, G. \& Joubert, D. (1999). Phys. Rev. B, 59, 1758-1775.

Ksenofontov, V., Wortmann, G., Chumakov, A. I., Gasi, T., Medvedev, S., McQueen, T. M., Cava, R. J. \& Felser, C. (2010). Phys. Rev. B, 81, 184510.

Liermann, H.-P., Konôpková, Z., Morgenroth, W., Glazyrin, K., Bednarčik, J., McBride, E. E., Petitgirard, S., Delitz, J. T., Wendt, M., Bican, Y., Ehnes, A., Schwark, I., Rothkirch, A., Tischer, M., Heuer, J., Schulte-Schrepping, H., Kracht, T. \& Franz, H. (2015). J. Synchrotron Rad. 22, 908-924.

Lindqvist, O., Haaland, A., Nilsson, J. E., Gronowitz, S., Christiansen, H. \& Rosén, U. (1968). Acta Chem. Scand. 22, 977-982.

Liu, L. G. (1987). J. Phys. Chem. Solids, 48, 719-722.

Matsunaga, T., Yamada, N., Kojima, R., Shamoto, S., Sato, M., Tanida, H., Uruga, T., Kohara, S., Takata, M., Zalden, P., Bruns, G., Sergueev, I., Wille, H. C., Hermann, R. P. \& Wuttig, M. (2011). Adv. Funct. Mater. 21, 2232-2239.

Mizuguchi, Y. \& Takano, Y. (2010). J. Phys. Soc. Jpn, 79, 102001.

Ogi, H., Fukunaga, M., Hirao, M. \& Ledbetter, H. (2004). Phys. Rev. $B, \mathbf{6 9}, 024104$.
Parshin, P. P., Chumakov, A. I., Alekseev, P. A., Nemkovski, K. S., Perßon, J., Dubrovinsky, L., Kantor, A. \& Rüffer, R. (2016). Phys. Rev. B, 93, 081102.

Perdew, J. P., Ruzsinszky, A., Csonka, G. I., Vydrov, O. A., Scuseria, G. E., Constantin, L. A., Zhou, X. \& Burke, K. (2008). Phys. Rev. Lett. 100, 136406.

Sergueev, I., Glazyrin, K., Kantor, I., McGuire, M. A., Chumakov, A. I., Klobes, B., Sales, B. C. \& Hermann, R. P. (2015). Phys. Rev. B, 91, 224304.

Sergueev, I., Wille, H.-C., Hermann, R. P., Bessas, D., Shvyd'ko, Y. V., Zając, M. \& Rüffer, R. (2011). J. Synchrotron Rad. 18, 802-810.

Seto, M., Yoda, Y., Kikuta, S., Zhang, X. W. \& Ando, M. (1995). Phys. Rev. Lett. 74, 3828-3831.

Shvyd'ko, Y. \& Gerdau, E. (1999). Hyperfine Interact. 123/124, 741776.

Simon, R. E., Sergueev, I., Kantor, I., Kantor, A., Perßon, J. \& Hermann, R. P. (2014). Semicond. Sci. Technol. 29, 124001.

Snyder, G. J. \& Toberer, E. S. (2008). Nat. Mater. 7, 105-114.

Sturhahn, W., Toellner, T. S., Alp, E. E., Zhang, X., Ando, M., Yoda, Y., Kikuta, S., Seto, M., Kimball, C. W. \& Dabrowski, B. (1995). Phys. Rev. Lett. 74, 3832-3835.

Thomas, P. A. (1988). J. Phys. C Solid State Phys. 21, 4611-4627.

Togo, A. \& Tanaka, I. (2015). Scr. Mater. 108, 1-5.

Wille, H.-C., Franz, H., Röhlsberger, R., Caliebe, W. A. \& Dill, F.-U. (2010b). J. Phys. Conf. Ser. 217, 012008.

Wille, H.-C., Hermann, R. P., Sergueev, I., Pelzer, U., Möchel, A., Claudio, T., Perßon, J., Rüffer, R., Said, A. \& Shvyd'ko, Yu. V. (2010a). Europhys. Lett. 91, 62001.

Wille, H. C., Shvyd'ko, Y. V., Alp, E. E., Rüter, H. D., Leupold, O., Sergueev, I., Rüffer, R., Barla, A. \& Sanchez, J. P. (2006). Europhys. Lett. 74, 170-176.

Worlton, T. G. \& Beyerlein, R. A. (1975). Phys. Rev. B, 12, 1899-1907. Wuttig, M. \& Yamada, N. (2007). Nat. Mater. 6, 824-832.

Zhang, D., Jackson, J. M., Chen, B., Sturhahn, W., Zhao, J., Yan, J. \& Caracas, R. (2013). J. Geophys. Res. Solid Earth, 118, 4071-4082.

Zhao, J., Sturhahn, W., Lin, J., Shen, G., Alp, E. E. \& Mao, H. (2004). High. Press. Res. 24, 447-457.

Zhao, J. Y., Bi, W., Sinogeikin, S., Hu, M. Y., Alp, E. E., Wang, X. C., Jin, C. Q. \& Lin, J. F. (2017). Rev. Sci. Instrum. 88, 125109. 